\title{
Quantitative Analysis of Nursing Observation Employing a Portable Eye-Tracker
}

\author{
Norie Suetsugu ${ }^{*}$, Masafumi Ohki², Tsunehisa Kaku² \\ ${ }^{1}$ Department of Nursing, Faculty of Medicine, Saga University, Saga, Japan \\ ${ }^{2}$ Department of Health Sciences, Graduate School of Medical Sciences, Kyushu University, Fukuoka, Japan \\ Email: "sue43@cc.saga-u.ac.jp, ohki@med.kyushu-u.ac.jp, kakut@med.kyushu-u.ac.jp
}

Received 24 December 2015; accepted 26 January 2016; published 29 January 2016

Copyright (C) 2016 by authors and Scientific Research Publishing Inc. This work is licensed under the Creative Commons Attribution International License (CC BY). http://creativecommons.org/licenses/by/4.0/

(c) $\underset{\mathrm{EY}}{\mathrm{B}}$ Open Access

\section{Abstract}

Background: Observation is an important skill for making appropriate nursing decisions and engaging in good practice. However, experts' observation behavior and cognitive processes cannot be easily verbalized or documented in an objective and accurate manner. Quantitative analysis of the observation behavior of nurses with rich clinical experience will yield effective educational data for fostering and improving nursing students' observation skills. Objectives: To improve nursing assessment education, the differences in the information gathering processes between clinical nurses and nursing students were analyzed by using a portable eye-tracker. Design: An experimental study. Settings: The experiment was performed at a university in Japan. Participants: The participants were 11 clinical nurses with at least 5 years of clinical experience for postoperative patients, and 10 fourth-year nursing students. Methods: In a mock hospital room, wherein we recreated a situation where a patient in postoperative day 1 was confined to a bed, participants wore an eye-tracking camera and engaged in nursing observation to make an early postoperative ambulation assessment of the patient. Participants' gaze points and gaze fixation durations were extracted from the gaze measurement data and compared. Results: Clinical nurses had shorter observation times and gaze durations than did nursing students, and focused more on the patient chart, intravenous drip, and indwelling drain. Students gazed for longest at the measuring devices for vital signs. Conclusions: We quantitatively analyzed differences in nursing observation according to clinical experience. Although no significant difference was found in gaze points, nursing students had a greater tendency to focus on information that was numerically displayed. Nurses with clinical experience conducted observations by gazing at information that they needed to focus on the most according to the patients' postoperative course.

"Corresponding author. 


\section{Keywords}

\section{Nursing Observation, Eye-Tracker, Postoperative Ambulation Assessment, Quantitative Analysis}

\section{Introduction}

Observation is an important skill for making appropriate nursing decisions and engaging in good nursing practice. Nurses' observational skills are attained and refined through accumulating clinical experience. There are two primary paradigms in expertise research: analyzing the technical characteristics of experts with abundant experience, and comparing the technical characteristics of experts and non-experts. Even in the nursing field, there are many studies involving interviews with skilled nurses and analyzing subjective data in order to elucidate such nurses' clinical decision-making process. However, experts' observation behavior and cognitive processes cannot be easily verbalized or documented in an objective and accurate manner. Observation is an action conducted through the five senses. For humans, out of the five senses, most information is gathered via the sense of sight. For instance, gaze is used for selectively gathering information [1]. As such, gaze measurement methods using eye-tracking cameras are believed to be effective means of obtaining detailed and objective assessments of human behavior or decision-making processes. From the late 1970s onward, research studies employing eye-tracking instruments have been conducted in the fields of cognitive psychology, pedagogy, and human engineering in order to elucidate the characteristics and decision-making processes involved in linguistic acquisition, ocular movement while driving, and visual searches of skilled technicians [2]-[5]. In recent years, it has become easier to measure ocular movements via miniature, lightweight mobile eye-tracking cameras that do not restrict observers' behavior [6]. From the 1990s onward, nursing studies have determined the efficacy of ocular counter-rolling instruments in the analysis of behavior to prevent accidents during drug administration and in providing feedback during simulation education in emergency nursing [7] [8]. However, there appears to be no similar studies on nurse expertise. This led us to the notion of quantitatively analyzing observational skills, which are fundamental to nursing practice, by tracking nurses' gazes in a setting that involves making nursing judgments. This would allow us to clarify the differences in observation skills between expert and novice nurses. In order to elucidate experts' visual search patterns, it would be desirable to employ an experimental setting that resembles the actual nursing practice environment. Employing eye-tracking instruments would enable us to objectively identify the characteristics of observational skills and compare them among different levels of clinical experience. This would involve measuring a series of nurse observation behaviors with an eye-tracking camera equipped while nurses act freely in an environment simulating a clinical setting.

This study aimed to determine the difference in nursing observation characteristics depending on the existence of clinical experience. Specifically, we compared the eye-tracking data of clinical nurses (experts) and nursing students (novices) during nursing observation to make an early postoperative ambulation assessment of a postoperative day 1 patient. Quantitatively analyzing the observation behavior of nurses with rich clinical experience will yield effective educational data for fostering and improving nursing students' observation skills.

\section{Methods}

\subsection{Participants}

Clinical nurses selected as participants for this study were at least level 4 (proficient) or higher according to Benner's 5-stage nursing skill levels [9]. The participants for this study were 12 nurses that worked at a university hospital and who had 5 or more years of clinical experience, of which 3 or more years were in the surgical field. The average clinical experience of nurses was 10.6 years (range: 6 - 25 years). Nursing students comprised 11 fourth-year university students (average age: 21 years) who had engaged in surgical nursing practice within their fundamental nursing education. All study participants provided informed consent, and each one signed for approval at this study. The study design was also approved by ethics review boards of Saga University and Kyushu University.

\subsection{Eye-Tracker}

For gaze measurement, we used the Talk Eye Lite eye tracker, manufactured by Takei Scientific Instruments Co., 
Ltd. This device comprises a goggle-type eye-tracking camera and miniature lightweight control unit worn on the waist and utilizes a computer to record data. The ocular movement measurement mechanism involves a central pupillary image processing method wherein weak infrared rays are cast at the cornea to generate a reflected image of the light source, and the location of this image is then detected. The sampling frequency is $30 \mathrm{~Hz}$, with the angle detection being 50 degrees horizontally and 20 degrees vertically. The data items detected are the direction of the gaze, gaze duration, gaze movement speed, pupil diameter, and the presence of blinking. It has been found that wearing glasses or soft contact lenses has virtually no impact on measurement.

\subsection{Simulation of Nursing Observation}

In a mock hospital room that recreated the situation of a patient being confined to a bed, clinical nurses and nursing students were asked to engage in observation to make an ambulation assessment of a patient in postoperative day 1.

Patient was played by the simulated patient who had received training. The vital signs of the patient actor were measured immediately before the experiment and it was confirmed that they showed no health abnormalities. Patients were instructed to not speak to the nurses or students during the observation experiment save to answer questions simply and exhibit anguished expressions as if in pain.

Figure 1 shows a participant equipped with an eye-tracking camera conducting a nursing observation. The simulated situation involved a patient recovering from a video-assisted thoracic surgery on right lung conducted the previous day; the patient was confined to the bed and was awake. Patients received oxygen via a mask, continuous intravenous drip infusion, and continuous intravenous infusion of epidural anesthesia. An indwelling urinary catheter was implanted in the patient's body while a thoracostomy tube was implanted in the patient's right thorax. The thoracostomy tube was attached to continuous low-pressure suction units and was in the process of drainage during the observation period. The mock hospital room had the patient's postoperative course record, and there was blood within the drain bag and urine in the urine drainage bag; devices displayed vital signs and emission volume indicating gradual recovery.

Participants were presented with the patient record, including the surgery performed before the experiment, both in writing and verbally. Participants entered the mock hospital room after being equipped with the goggle-type eye-tracking camera and undergoing equipment calibration to ensure that it accurately recorded their gazes. The tracking and recording of participants' ocular movement using the eye-tracking camera commenced as soon as they entered the room. No time restriction for making the ambulation assessment was given; the observation was regarded as complete when the participant gave their judgment.

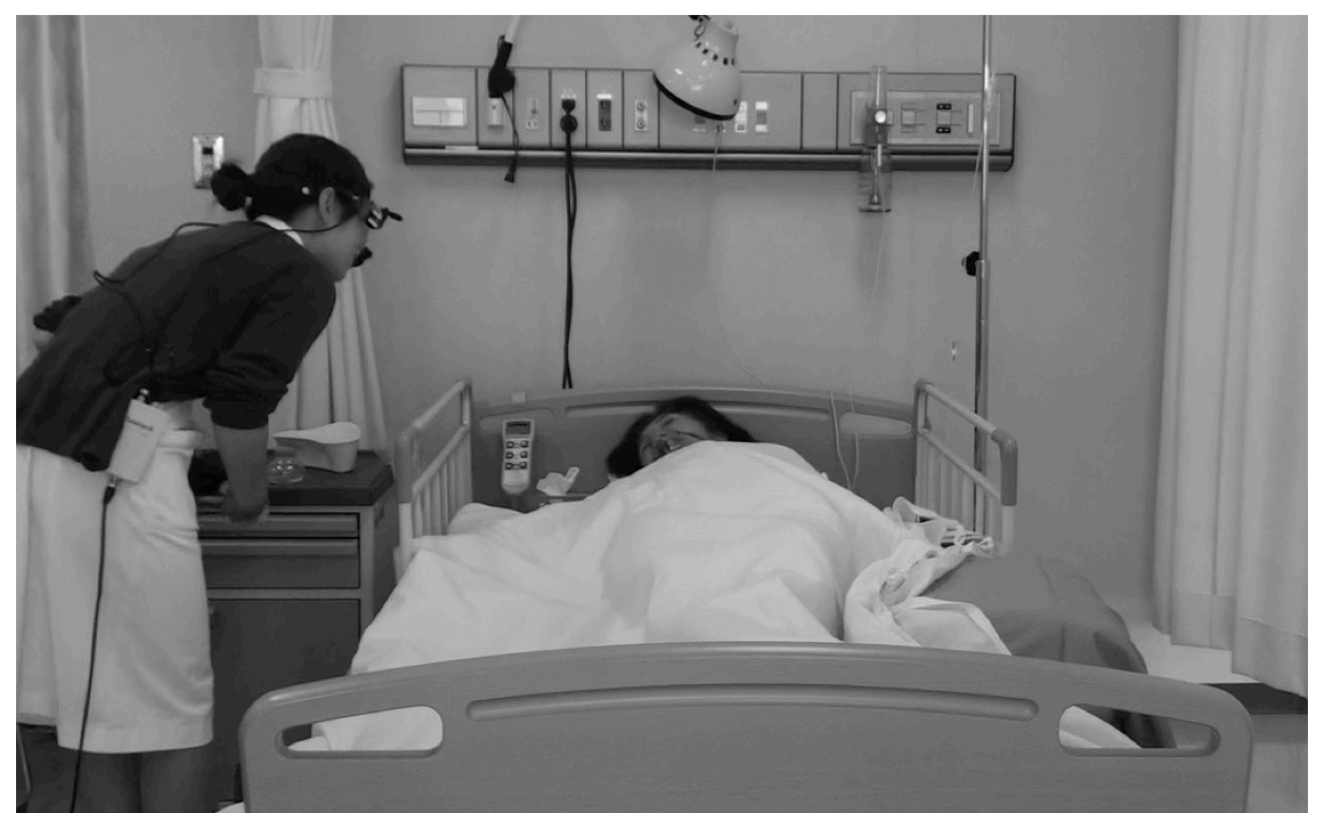

Figure 1. Simulation of nursing observation. 


\subsection{Data Analysis}

A gaze was defined as a state in which a line of sight was sustained for over 150 milliseconds with the ocular movement angle change being less than 5 degrees per second. After the completion of the observation, the gaze points and the total gaze duration of these points were calculated from the eye-tracking data of clinical nurses and nurse students. The difference in gaze duration between nurses and students was compared with the MannWhitney U test using the statistical analysis software JMP (ver. 11). The p-value less than 5\% was considered statistically significant.

\section{Results}

Since obvious measurement errors were found in the data of one clinical nurse and one nursing student, these two participants were excluded from further analysis. Thus, the results of 11 nurses and 10 students were analyzed. Nurses and students that completed the nursing observation all determined that the patient could leave the bed. The average and one standard deviation of observation time required to complete the assessment was 7 mins 21 secs \pm 1 min 59 secs for nurses and 13 mins 48 secs \pm 5 min 3 secs for students. Students spent a significantly longer time $(\mathrm{p}<0.001)$.

Figure 2 shows the differences in gaze points and gaze movement between students and nurses when inspecting patients' faces. The students tended to gaze at the periphery of gaze points (e.g., the patient's face) by slightly changing the position of their gaze and showed considerable gaze movement. Meanwhile, nurses showed fewer gaze points and less gaze movement.

We extracted the points at which one participant gazed at least from the beginning to the end of the observation period, revealing 18 gaze points overall as shown in Table 1. These points were the patient's face, chest, body (except chest), clinical thermometer, manometer, stethoscope, pulse oximeter, wristwatch, patient chart, memo pad, gauze bandage, indwelling drain, continuous aspirator, intravenous drip, epidural anesthesia kit, urine bag, bed, and bedding.

Figure 3 shows a comparison of nurses and students in the percentage of participants who gazed at each point. The points at which over $50 \%$ of both nurses and students gazed were the face, stethoscope, manometer, and pulse oximeter. The points at which there were twice as many or more nurses than students gazing were the $p a-$ tient record, intravenous drip, and indwelling drain. Conversely, the points for which the percentage of students was twice or more that of the nurses were the body (except chest), clinical thermometer, wristwatch, memo pad, and bed. Notably, none of the nurses gazed at the wristwatch while 7 out of 10 students did.

Figure 4 shows a comparison of gaze duration at each point between nurses and students using box plots. The white lines in the boxes are the median values while the lower and upper limits represent minimum and maximum values, respectively. The points at which we noted statistically significant differences in gaze duration between nurses and students were the body (except chest) $(\mathrm{p}=0.048)$ and the memo pad $(\mathrm{p}=0.003)$. At both points, the gaze duration of the students was longer. For wristwatch, one student gazed at it at most for 23 seconds. Although no statistically significant difference was found, the points at which the average gaze duration of the nurses was longer than was that of the students by three times or more were patient record, intravenous

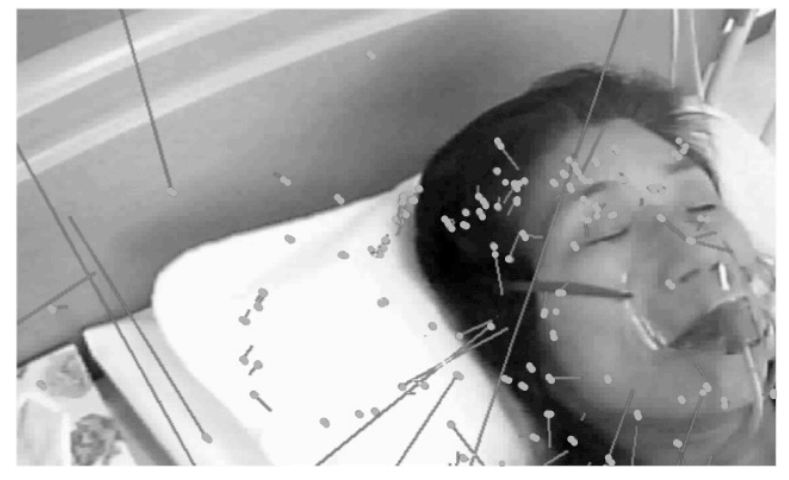

(a)

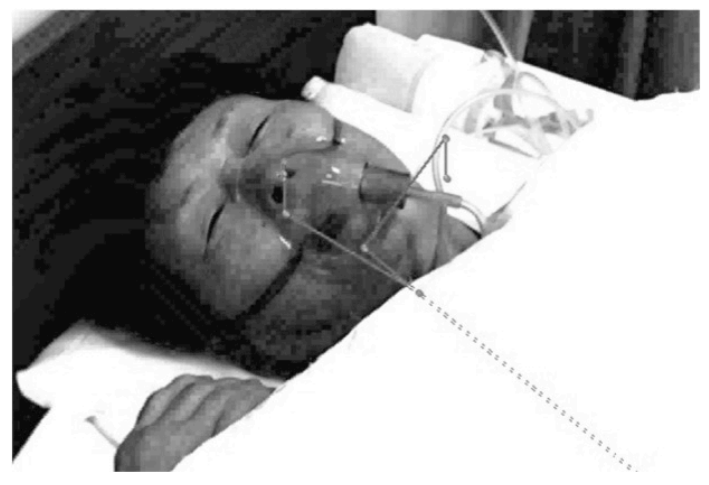

(b)

Figure 2. Gaze points and eye movements (a) Nursing student's view, (b) clinical nurse's view. Dots and lines represent gaze points and eye movements, respectively. 
Table 1. Gaze points and durations.

\begin{tabular}{|c|c|c|c|c|c|c|c|c|c|}
\hline \multirow{3}{*}{$\begin{array}{c}\text { Gaze } \\
\text { object }\end{array}$} & \multirow{2}{*}{ Gaze point } & \multicolumn{2}{|c|}{$\begin{array}{c}\text { Percentage gazed } \\
(\%)\end{array}$} & \multicolumn{2}{|c|}{$\begin{array}{l}\text { Medium gaze duration } \\
\text { (sec) }\end{array}$} & \multicolumn{2}{|c|}{$\begin{array}{l}\text { Maximum gaze duration } \\
\text { (sec) }\end{array}$} & \multicolumn{2}{|c|}{$\begin{array}{l}\text { Average gaze duration } \\
\text { (sec) }\end{array}$} \\
\hline & & \multicolumn{2}{|c|}{ Nurse student } & \multicolumn{2}{|c|}{ Nurse student } & \multicolumn{2}{|c|}{ Nurse student } & \multicolumn{2}{|c|}{ Nurse student } \\
\hline & Face & 73 & 100 & 1.7 & 1.5 & 4.3 & 8.0 & 1.59 & 2.04 \\
\hline \multirow[t]{4}{*}{ Patient } & Chest & 27 & 30 & 0.0 & 0.0 & 0.3 & 13.5 & 0.06 & 1.40 \\
\hline & Body (except chest) & 18 & 60 & 0.0 & 0.3 & 0.8 & 1.4 & 0.09 & 0.39 \\
\hline & Clinical thermometer & 18 & 40 & 0.0 & 0.0 & 0.4 & 2.6 & 0.07 & 0.39 \\
\hline & Stethoscope & 64 & 90 & 0.5 & 1.1 & 7.9 & 3.3 & 1.53 & 1.45 \\
\hline \multirow[t]{3}{*}{ Devices } & Manometer & 91 & 90 & 3.1 & 0.7 & 5.9 & 13.3 & 2.78 & 2.72 \\
\hline & Pulse oximeter & 82 & 70 & 0.6 & 1.1 & 5.9 & 9.5 & 0.97 & 2.41 \\
\hline & Wristwatch & 0 & 70 & 0.0 & 1.3 & 0.0 & 23.2 & 0.00 & 4.64 \\
\hline \multirow{2}{*}{ Records } & Patient chart & 36 & 10 & 0.0 & 0.0 & 1.5 & 0.2 & 0.20 & 0.02 \\
\hline & Memo pad & 27 & 80 & 0.0 & 1.0 & 0.4 & 10.0 & 0.07 & 2.38 \\
\hline \multirow{6}{*}{ Catheters } & Intravenous drip & 36 & 10 & 0.0 & 0.0 & 0.2 & 0.2 & 0.06 & 0.02 \\
\hline & Gauze bandage & 9 & 20 & 0.0 & 0.0 & 0.2 & 0.2 & 0.02 & 0.04 \\
\hline & Indwelling drain & 45 & 10 & 0.0 & 0.0 & 1.1 & 0.2 & 0.16 & 0.02 \\
\hline & Continuous aspirator & 27 & 40 & 0.0 & 0.0 & 0.3 & 1.6 & 0.06 & 0.29 \\
\hline & $\begin{array}{l}\text { Epidural anesthesia } \\
\text { kit }\end{array}$ & 18 & 30 & 0.0 & 0.0 & 1.4 & 0.8 & 0.23 & 0.15 \\
\hline & Urine bag & 18 & 20 & 0.0 & 0.0 & 0.4 & 1.4 & 0.06 & 0.21 \\
\hline \multirow{2}{*}{ Others } & Bed & 9 & 20 & 0.0 & 0.0 & 0.2 & 2.7 & 0.02 & 0.31 \\
\hline & Bedding & 2 & 30 & 0.0 & 0.0 & 0.2 & 2.1 & 0.04 & 0.37 \\
\hline
\end{tabular}

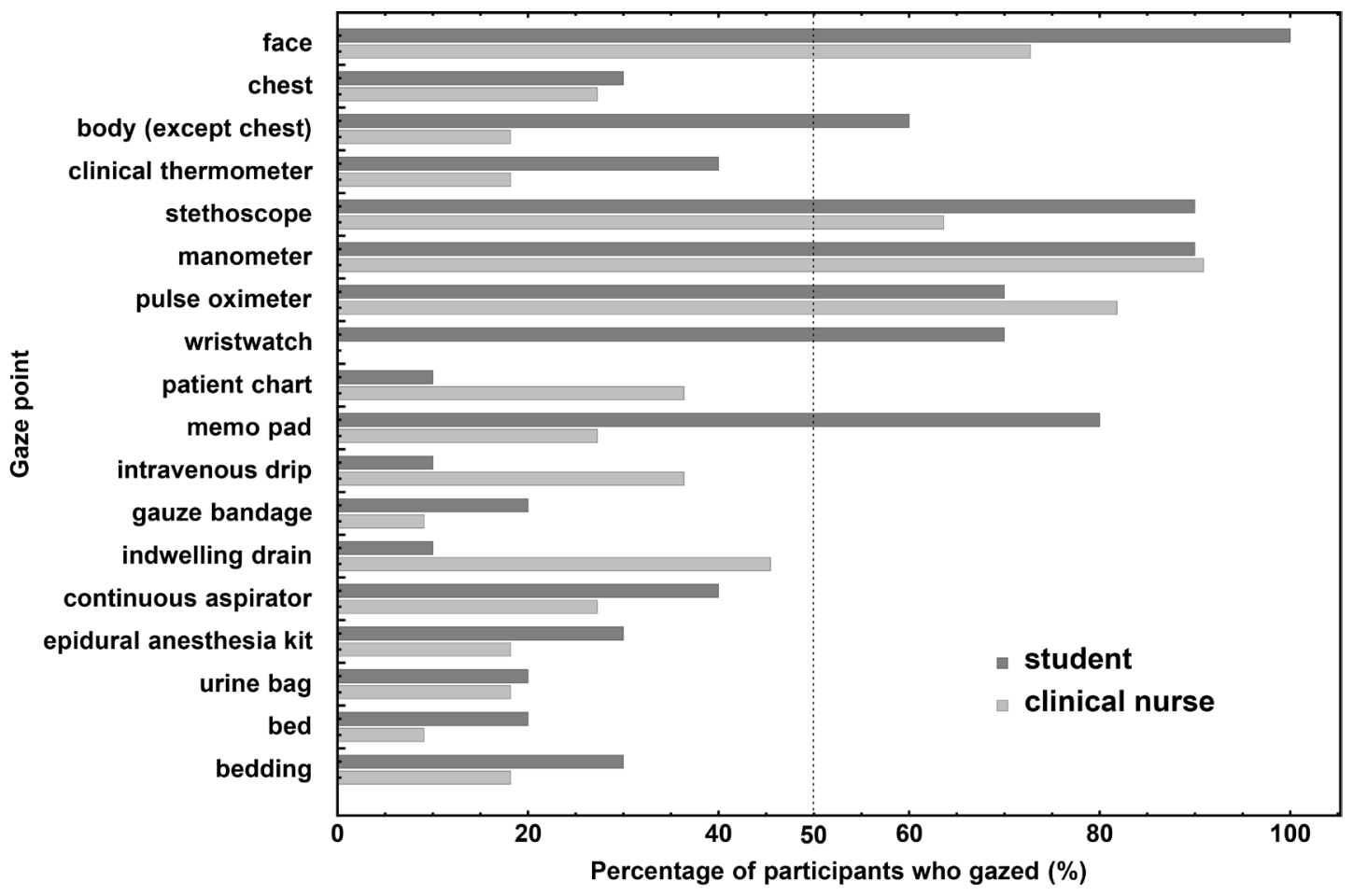

Figure 3. Percentage of participants who gazed at the 18 gaze points. 


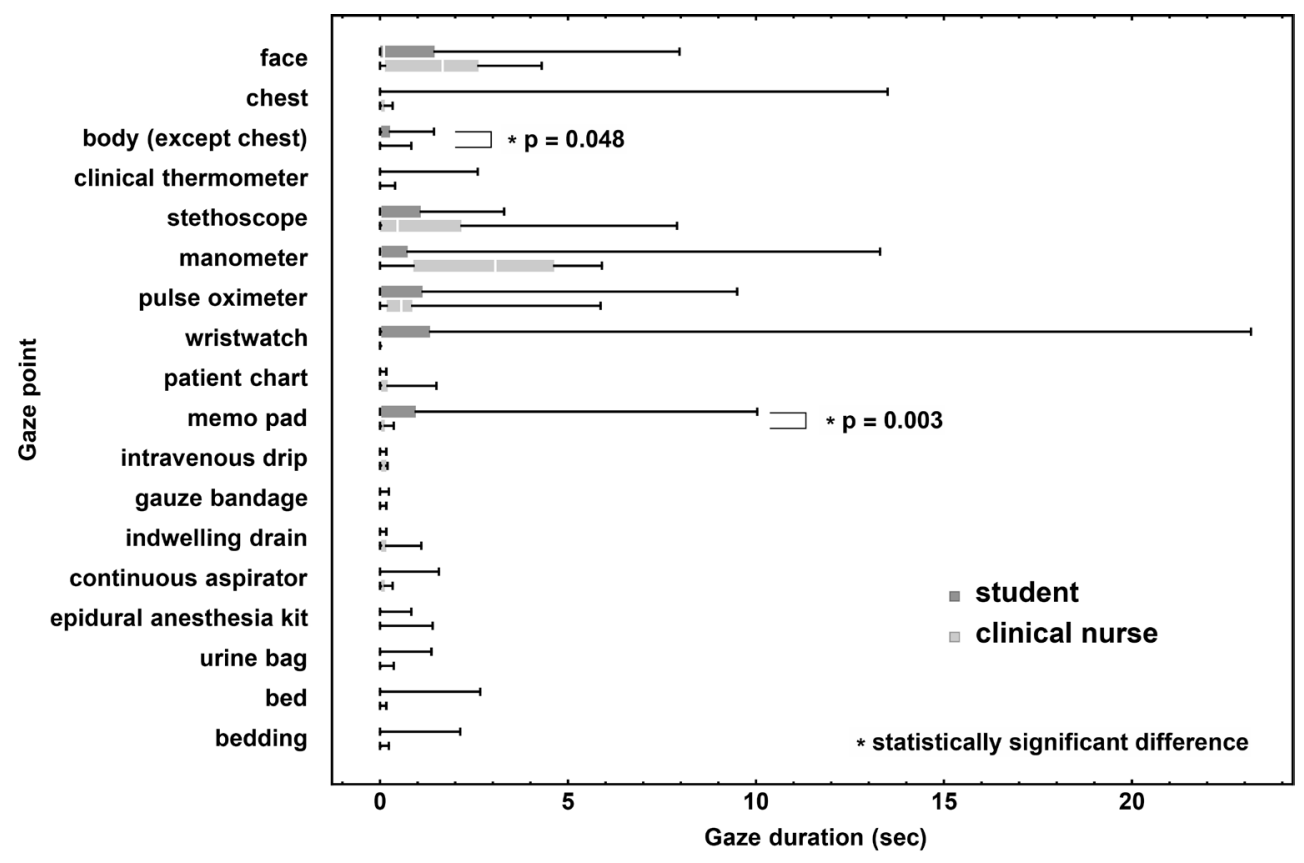

Figure 4. Gaze duration at the 18 gaze points.

drip, and indwelling drain. Regarding vital sign measurement devices, the median gaze duration was longer for students than for nurses for the stethoscope and pulse oximeter. The median gaze duration for the manometer was longer for the nurses than for the students.

The 18 gaze points were classified into 5 different categories of patient, vital sign measurement devices, records, catheters, and others as shown in Table 1. Figure 5 displays that integrated gaze duration for each category in a boxplot. Of the 5 categories, the vital sign measurement devices had the longest gaze duration for both nurses and students and showed a significant difference from other categories. The category with the second longest gaze duration was patient; when comparing the patient and records categories, there was no significant difference for students but there was one for nurses $(\mathrm{p}=0.03)$. Only the records showed a significant difference between nurses and students, with students having longer gaze durations $(p=0.03)$.

\section{Discussion}

This study showed that clinical nurses spent at least half the time than did nursing students in performing observations to obtain an early postoperative ambulation assessment. The nursing observation was performed according to an objective: namely, to determine whether a postoperative day 1 patient can leave their bed or not in this study. Early postoperative ambulation is an important nursing assistance for preventing postoperative complications. The necessary points to observe for making an accurate ambulation assessment are vital signs, the patient's physical condition (judged from preoperative strength and operative stress), the degree of pain, potential risks of collapsing or symptom deterioration, and the patient's volition [10]. The participating nurses in this study all had over 6 years of clinical experience in the surgical field and had conducted ambulation assessments of postoperative patients. Because of this background, they were able to identify specific observation points necessary for making an accurate judgment and found it easy to make decisions because the mock situation and simulated patient were similar to situations they had experienced in the past where patients were able to leave the bed. These factors are believed to have led nurses to complete the observation within a shorter period than the students [11] [12]. However, it is believed that the time required to make a judgment in an actual clinical setting would be shorter than that found in the present study because the nurses would preoperatively know the patient's physical and mental background information. Students, compared to nurses, had larger gaze movement, more gaze points, and spent more time gazing at each point because they had to interpret each piece of information in a serial manner. It is believed that these factors extended students' observation duration.

The gaze point refers to an area in which the observer showed interest. The points at which most participants 


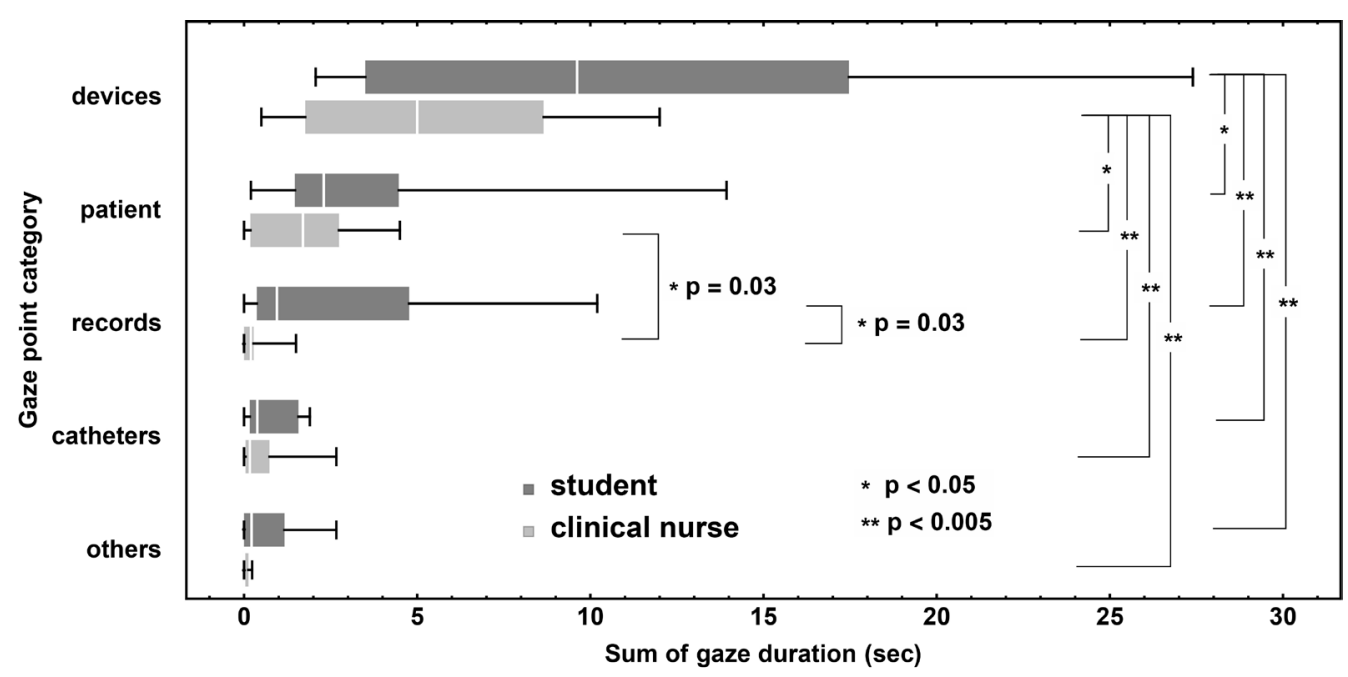

Figure 5. Gaze duration at the five gaze point categories.

gazed were the face, stethoscope, manometer, and pulse oximeter, indicating that they gazed at the patient's expression and vital sign measurement devices. In this observation experiment, participants had to rely greatly on their visual perception for the information needed to make a judgment because we had the patient show expressions of pain while limiting their verbal statements and had actual operating medical equipment and drip infusions. As shown in Figure 2, while there was a clear difference in gaze movement between nurses and students, there were no significant differences in the gaze points necessary for making an ambulation assessment. Concerning the wristwatch, while students gazed at it, no nurses did so; this is because the students palpated the pulse by following basic procedures-namely, counting the number of breaths by checking the patient's chest movements and gazing at their own wristwatches while measuring. In contrast, nurses checked pulse count using the numerical value displayed on the pulse oximeter; in other words, they used medical equipment. However, pulse oximeters, which measure the pulse of arterial blood and oxygen saturation by issuing a light beam onto the skin, lose accuracy when peripheral blood is low, the probe experiences an attachment failure, or when there is too much ambient light [13]. Thus, it might be necessary to palpate the pulses in accordance with the basic procedure as the students did in order to ensure an accurate nursing diagnosis. The points at which at least twice as many students as nurses gazed were the body (except chest), clinical thermometer, memo pad, and bed. It is believed that students gazed at the body (except chest) and bed because they were taught to do so as part of nursing observation basics during their fundamental nursing education. As for the memo pad, $80 \%$ of the students gazed at it to record vital signs data, and their gaze duration were significantly longer than was that of nurses. As mentioned above, it can be inferred that the students performed observations that were faithful to the basic procedures.

Compared to nurses, students' gaze duration on the chest was longer, suggesting that they required more time to determine the breathing state. In contrast, nurses likely quickly noted the breath count and rhythm while observing the patient's breathing, without gazing at the chest area. The points at which nurses spent a longer time gazing compared to students were the patient record, intravenous drip, and indwelling drain. The reasoning for this finding is that the nurses confirmed the successive course of the patient immediately after the surgery by looking at the patient record so that they could predict the patient's current state. It is believed that the nurses gathered information on patient safety by checking the indwelling drain and intravenous drip implanted in the patient. Bleeding is a symptom that cannot be overlooked in this simulated patient's situation. Thus, the drainage amount and properties from the indwelling drain is an important information source for assessing postoperative bleeding, which explains the longer duration of nurses' gazing at it [14]. On the other hand, the gaze duration for the continuous aspirator and the gauze bandage applied to the wounded area was longer among students, perhaps because they focused on the drainage accumulated in the drainage bottle of the continuous aspirator and were checking for bleeding on the gauze. In other words, nurses tended to focus on current bleeding while students tended to focus on overall bleeding volume during observations related to postoperative bleeding.

In Figure 5, the 18 gaze points were divided into 5 categories and compared. Under this classification, both 
the nurses and the students gazed at vital sign measurement devices significantly longer than they did the other 4 categories. This is believed to be because gazing at these devices was necessary to ascertain the measured vital signs. Although no significant difference in gaze duration between the patient and records categories was found for the students, there was a significant difference for the nurses, which indicates that they looked at the patient more than they did the records.

There are considered to be two ways of looking at the world: direct vision and peripheral vision. Direct vision occurs when an image is formed at the central area of the retina when consciously gazing at a single point. Peripheral vision, by contrast, occurs when the image forms on the peripheral area of the retina and reflects the peripheral area of a scene; it is a viewing method that comprehensively captures spatial positional relation and movement information [15]. The gaze duration measured in this study using an eye-tracking camera was the duration of direct vision. Peripheral vision is also considered to be important factor in actual nursing assessments conducted by sight [16] [17]. In addition, nurses who observe postoperative patients on a daily basis conduct observations by presupposing required information other than visual information in advance. Thus, it is believed that nurses make assessments by drawing on their accumulated knowledge during observations. Further evaluations of nursing assessment processes must consider these points.

Although nursing students took more than twice as long as nurses did in the observation period to make an assessment, they were still able to make a correct assessment concerning the patient's ambulation. This is believed to be because these nursing students were in their $4^{\text {th }}$ year of university, meaning that they knew the fundamentals of nursing care. Students (i.e., novices) gathered numerical information-mainly vital sign measurement data and bleeding volumes - and used it for conducting their nursing assessment. However, there are cases when no change occurs in vital sign values immediately after a physical abnormality occurs [18]. Thus, nursing assessment education for novices must foster skills that enable them to not only interpret numerical information, but also be able to link this with other information in the situation. The quantitative analysis of nursing observation behavior in this study visualized the relationships between the information in the scene and aided in elucidating the nursing assessment process.

\section{Conclusion}

Differences in nursing observation behavior according to the existence of clinical experience were analyzed quantitatively in this study. Although no significant difference was found in terms of gaze points, the nursing students had a tendency to focus on information that could be easily represented numerically. We also found that nurses with clinical experience conducted observations by gazing at the information that they needed to focus on most at any given time according to the patients' postoperative course.

\section{Funding}

The study was supported by a grant from the Japan Society for the Promotion of Science (KAKENHI 15 K11469). The authors have no conflicts of interest to declare. All study participants provided informed consent, and the study design was approved by ethics review boards of Saga University (No. 26 - 40) and Kyushu University (No. 27 - 84).

\section{References}

[1] Rayner, K. (1998) Eye Movements in Reading and Information Processing: 20 Years of Research. Psychological Bulletin, 124, 372-422. http://dx.doi.org/10.1037/0033-2909.124.3.372

[2] Just, M.A. and Carpenter, P.A. (1980) A Theory of Reading: From Eye Fixations to Comprehension. Psychological Review, 87, 329-354. http://dx.doi.org/10.1037/0033-295X.87.4.329

[3] Krajbich, I., Amel, C. and Rangel, A. (2010) Visual Fixations and the Computation and Comparison of Value in Simple Choice. Nature Neuroscience, 13, 1292-1298. http://dx.doi.org/10.1038/nn.2635

[4] Tole, J.R., Stephens, A.T., Harris, R.L.Sr. and Ephrath, A.R. (1982) Visual Scanning Behavior and Mental Workload in Aircraft Pilots. Aviation Space and Environmental Medicine, 53, 54-61.

[5] Underwood, G. (2007) Visual Attention and the Transition from Novice to Advanced Driver. Ergonomics, 50, 12351249. http://dx.doi.org/10.1080/00140130701318707

[6] Mele, M.L. and Federici, S. (2012) Gaze and Eye-Tracking Solutions for Psychological Research. Cognitive Processing, 


\section{3, 262-265. http://dx.doi.org/10.1007/s10339-012-0499-z}

[7] Marquard, J.L., Junghee, J., Henneman, P.L., Fisher, D.L. and Henneman, E.A. (2013) Can Visualizations Complement Quantitative Process Analysis Measures? A Case Study of Nurses Identifying Patients before Administering Medications. Journal of Cognitive Engineering and Decision Making, 7, 198-210. http://dx.doi.org/10.1177/1555343412457683

[8] O’Meara, P., Munro, G., Williams, B., Cooper, S., Bogossian, F., Ross, L., Sparkes, L., Browning, M. and McClounan, M. (2015) Developing Situation Awareness amongst Nursing and Paramedicine Students Utilizing Eye Tracking Technology and Video Debriefing Techniques: A Proof of Concept Paper. International Emergency Nursing, 23, 94-99. http://dx.doi.org/10.1016/j.ienj.2014.11.001

[9] Benner, P. (2001) From Novice to Expert: Excellence and Power in Clinical Nursing Practice. Prentice-Hall, Upper Saddle River.

[10] Zeitz, K. (2005) Nursing Observations during the first 24 Hours after a Surgical Procedure: What Do We Do? Journal of Clinical Nursing, 14, 334-343. http://dx.doi.org/10.1111/j.1365-2702.2004.01071.x

[11] Tanner, C.A. (2006) Thinking Like a Nurse: A Research-Based Model of Clinical Judgment in Nursing. Journal of Nursing Education, 45, 204-211.

[12] Forsberg, E., Zieqert, K., Hult, H. and Fors, U. (2013) Clinical Reasoning in Nursing, a Think-Aloud Study Using Virtual Patients-A Base for an Innovative Assessment. Nurse Education Today, 34, 538-542. http://dx.doi.org/10.1016/j.nedt.2013.07.010

[13] Yelderman, M. and New, Jr., W. (1983) Evaluation of Pulse Oximetry. Anesthesiology, 59, 349-352. http://dx.doi.org/10.1097/00000542-198310000-00015

[14] Hoffman, K.A., Aitken, L.M. and Duffield, C. (2009) A Comparision of Novice and Expert Nurses’ Cue Collection during Clinical Decision-Making Verbal Protocol Analysis. International Journal of Nursing Studies, 46, 1335-1344. http://dx.doi.org/10.1016/j.ijnurstu.2009.04.001

[15] Sardegna, J., Shelly, S., Rutzen, A.R. and Scott, M.S. (2002) The Encyclopedia of Blindness and Vision Impairment. 2nd Edition, Infobase Publishing, New York.

[16] Hayhoe, M. and Ballard, D. (2005) Eye Movements in Natural Behavior. Trends in Cognitive Sciences, 9, $188-194$. http://dx.doi.org/10.1016/j.tics.2005.02.009

[17] Yarbus, A.L. (1967) Eye Movements and Vision. Plenum Press, New York. http://dx.doi.org/10.1007/978-1-4899-5379-7

[18] Brier, J., Carolyn, M., Haverly, M., Januario, E., Padula, C., Tal, A. and Triosh, H. (2014) Knowing “Something Is Not Right” Is Beyond Intuition: Development of a Clinical Algorithm to Enhance Surveillance and Assist Nurses to Organise and Communicate Clinical Findings. Journal of Clinical Nursing, 24, 832-843. http://dx.doi.org/10.1111/jocn.12670 